\title{
INFLUÊNCIA DOS FATORES AMBIENTAIS NA \\ ABUNDÂNCIA DE PORTUNUS SPINIMANUS LATREILLE, \\ 1819 (CRUSTACEA, BRACHYURA, PORTUNIDAE) NA \\ REGIÃO DE UBATUBA (SP)
}

Sandro Santos

Departamento de Biologia -CCNE -ssantos@ccne.ufsm.br

UFSM - Santa Maria, RS

\section{ABSTRACT}

This work aimed to study the environmental factors influence on $P$. spinimanus abundance in the sublittoral sediments of Fortaleza bay, Ubatuba (SP). The collects were made during one year, from November/1988 to October/1989, with a shrimp fishery boat equipped with 2 otter-trawl. Temperature, salinity, depth, dissolved oxygen content, organic material amount of the sediment and its granulometric composition were the environment factors sampled. The procedure adopted here consisted in to distribute the environment factors results in strata, to determine the values frequency and the number of captured individuals in each stratum through the 84 samples. The transformed abundance index was calculated by means the formula $A=\operatorname{Ln}(x+1)$, where, $x=$ mean number of individuals and $\operatorname{Ln}=$ neperian logarithm. The abundance index tended to increase with the temperature elevation and it was high in 31.6 to $33.2 \%$ of salinity. The abundance index for both dissolved oxygen and organic material contents 
were characterized by a normal curve. The individuals were abundant in the first two strata of depth. With relation to granulometric fractions, the animals were more abundant in subareas with gravel and very coarse sand than the others. Based on this results and field observations it can be concluded that the presence of $P$. spinimanus is associated to a group of environment factors among them the sediment texture (gravel and very coarse sand) and to protected sites in the bay, characterized by a relatively calm water.

Key words: Crustacea, Portunidae, environmental factors, Brazilian coast

\section{INTRODUÇÃO}

Padrões ambientais característicos podem proporcionar condições favoráveis para a efetivação de diversas funções vitais de um ser. Tais padrões representam estímulos capazes de desencadear processos como reprodução ou vantagens adaptativas às espécies. Quando se conhece as relações entre o ambiente e os organismos que nele vivem, torna-se possível diagnosticar de forma efetiva impactos ambientais e, no caso de regiões pesqueiras sujeitas a problemas de pesca predatória, proporcionar uma exploração racional dos recursos, sem o comprometimento dos estoques pesqueiros. $\mathrm{O}$ ambiente marinho apresenta fatores abióticos importantes que agem na abundância de organismos bentônicos, como a temperatura, a salinidade, o teor de oxigênio dissolvido na água, o sedimento, as correntes e, indiretamente, a profundidade.

A família Portunidae tem sido alvo de estudos sobre a distribuição das espécies associada aos fatores ambientais. Neste aspecto, os siris azuis Callinectes têm sido muito investigados talvez devido à sua abundância e importância econômica (TAISSOUN, 1973a). 
Para o gênero Portunus poucas são as referências a esse respeito, entre elas pode-se citar as de DHAWAN et al. (1976) e POTTER et al. (1983) que investigaram vários aspectos da biologia de $P$. pelagicus, em algumas regiões do globo, enfocando a associação dos fatores ambientais na distribuição, no desenvolvimento e na sua ecologia.

De acordo com MELO (1996), P. spinimanus ocorre no Atlântico ocidental desde New Jersey até o sul da Flórida, Golfo do México, América Central, Antilhas, Guianas e Brasil (de Pernambuco até o Rio grande do Sul). POWERS (1977) cita a presença desta espécie em braços de mar, canais e baías, com fundo de areia, cascalho conchífero e lodo, da região entre marés até 91 metros de profundidade.

O presente trabalho destina-se a abordar a biologia de $P$. spinimanus, como subsídio para o entendimento de alguns mecanismos adaptativos da espécie, quanto a sua abundância frente aos fatores ambientais temperatura, salinidade, teor de oxigênio dissolvido na água, textura do substrato e profundidade.

\section{MATERIAL E MÉTODOS}

Foram realizadas coletas mensais por um período de um ano (de novembro de 1988 a outubro de 1989) em 7 subáreas da Enseada da Fortaleza, Ubatuba, SP (figura 1).

As coletas foram feitas com um barco para a pesca de camarão (30 minutos de arrasto em cada área), equipado com duas redes do tipo "otter trawl", de 7,5 metros de comprimento cada uma, 15 milímetros entre-nós na panagem e 10 milímetros no saco, abertura da boca de 3,70 
metros de largura e 2,00 metros de altura. O saco de rede media 2,40 metros de comprimento e 0,75 metros de abertura.

Os caranguejos foram triados e acondicionados em sacos plásticos, etiquetados e colocados em caixas térmicas, com gelo picado, para o transporte até o laboratório de Carcinologia do Departamento de Zoologia do Instituto de Biociências da UNESP, "Campus" de Botucatu, onde foram conservados congelados até o momento das análises.

A cada arrasto, após as redes serem recolhidas, o barco retornava até o ponto médio, da estação correspondente, onde procedia-se a amostragem dos fatores ambientais.

A água do fundo foi coletada com auxílio de uma garrafa de NANSEN e a água da superfície, com um balde. Assim obteve-se amostras para o registro da temperatura, salinidade e teor de oxigênio dissolvido.

A temperatura foi medida com um termômetro de mercúrio $\left({ }^{\circ} \mathrm{C}\right)$ e a salinidade (\%) com um refratômetro específico.

Para a determinação do teor de oxigênio, foi sifonada água para frascos âmbar de $250 \mathrm{~mL}$, marcados com o número de cada estação. O teor de oxigênio foi calculado de acordo com o método titulométrico proposto por GOLTERMAN \& CLYMO (1969), modificado pela adição de azida sódica.

A medida da profundidade, de cada local amostrado, foi feita por meio da corda que estava presa ao pegador de VAN-VEEN, graduada de 50 em 50 centímetros.

O sedimento foi coletado com pegador de VAN-VEEN com $1 / 40 \mathrm{~m} 2$ de área de amostragem. Cerca de trezentos gramas foram transferidos para sacos plásticos, devidamente etiquetados e transportados até o laboratório em Botucatu (SP). As amostras de sedimento foram

colocadas em placas de Petri $(80 \mathrm{~mL})$ devidamente marcadas com o número da respectiva estação e deixadas em estufa a $70^{\circ} \mathrm{C}$, por 72 horas. 
Após a secagem, as amostras de sedimento foram divididas em subamostras, para análises da Matéria Orgânica e Granulometria. O teor de matéria orgânica foi obtido pelo peso das cinzas livres. A textura do sedimento foi obtida pelo peneiramento diferencial, baseando-se na escala americana (WENTHWORTH, 1922).

Após o descongelamento parcelado de cada amostra mensal iniciava-se a triagem, contagem e identificação geral dos braquiúros. Desse modo, todos os $P$. spinimanus foram separados e registrados. Cada indivíduo coletado foi etiquetado com um número e anotou-se também o sexo e estado de maturidade do animal, segundo TAISSOUN (1973b).

Os resultados das análises dos fatores ambientais foram correlacionados com a presença ou ausência de $P$. spinimanus

Tais correlações consistiram em distribuir os resultados obtidos para os fatores ambientais em classes e determinar o número de espécimes capturados e a freqüência de repetições dos valores dentro de cada classe, durante as amostragens efetuadas na enseada. A etapa seguinte foi calcular o índice de abundância transformada para cada classe, por meio da fórmula apresentada por HAEFNER (1976): Ln $(x+1)$; onde, $x=$ número médio de indivíduos e $\mathrm{Ln}=$ logaritmo neperiano. 


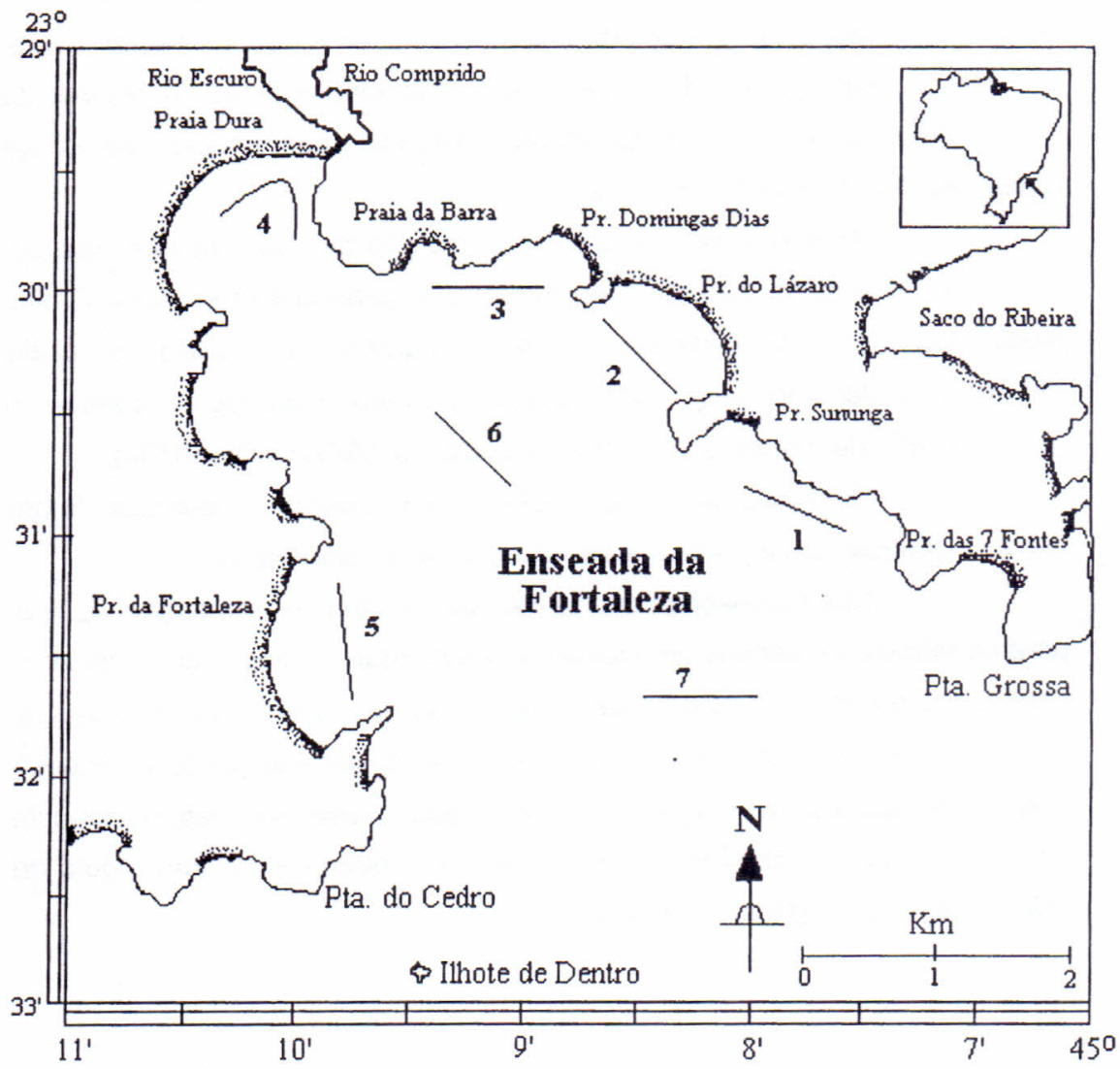

Figura 1: Localização das 7 subáreas de amostragem na Enseada da Fortaleza, Ubatuba (SP). 


\section{RESULTADOS}

Obteve-se um total de 126 indivíduos de $P$. spinimanus, dos quais 26 eram fêmeas adultas, 24 eram machos adultos e 76 indivíduos jovens.

Os valores médios dos fatores ambientais registrados estão contidos nas tabelas I e II.

Os resultados obtidos da distribuição da abundância transformada em classes dos fatores ambientais estão apresentados nas figuras 2 e 3 .

Quanto a temperatura, a abundância de $P$. spinimanus foi mais pronunciada na faixa de 28 a $30^{\circ} \mathrm{C}$ e quanto a salinidade, na faixa de 31,6 a $33,2 \%$ (figura 2). A associação de $P$. spinimanus com o teor de oxigênio dissolvido na água caracteriza uma curva normal, com um pico de tolerância ao oxigênio dissolvido na classe 3 (de 4,6 a 5,7 mg/L) e a matéria orgânica na classe 3 (de 5,0 a 7,5\%). O maior índice de abundância obtido para a associação com a profundidade ocorreu na classe 2 (de 6,0 a 8,5m).

Com relação à textura do sedimento, $P$. spinimanus apresenta uma distribuição normal nas associações com Areia Grossa, Areia Média e Areia Fina. Obteve-se uma relação inversa com as frações Areia Muito Fina e Silte-Argila. A fração Cascalho apresenta maiores índices de abundância para as classes 2 e 5 (de 5 a 10 e de 20 a $25 \%$ ). Estas associações podem ser observadas na figuras 3 . 

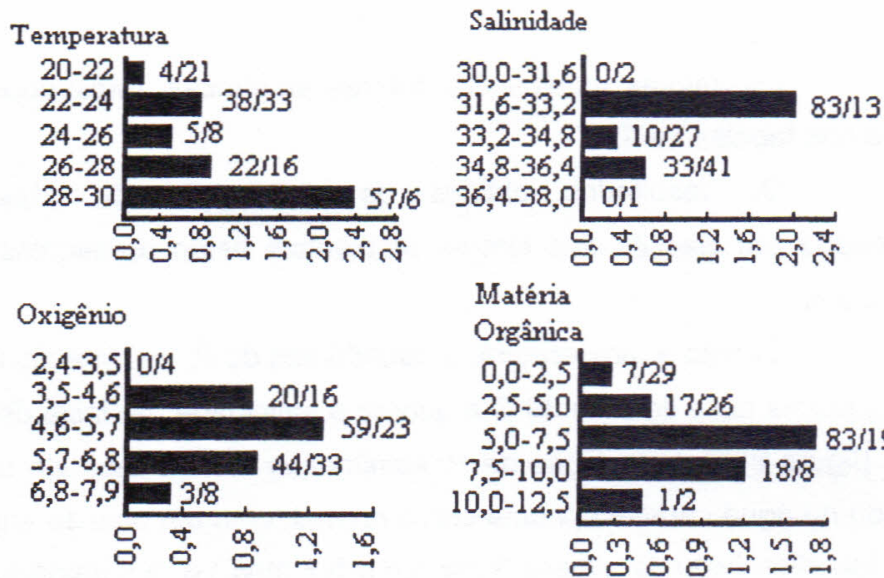

Matéria

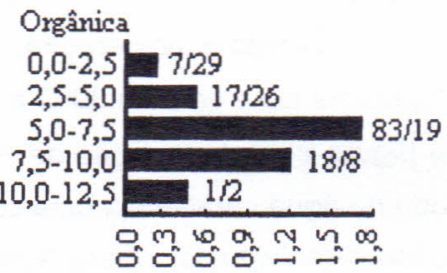

Profundidade

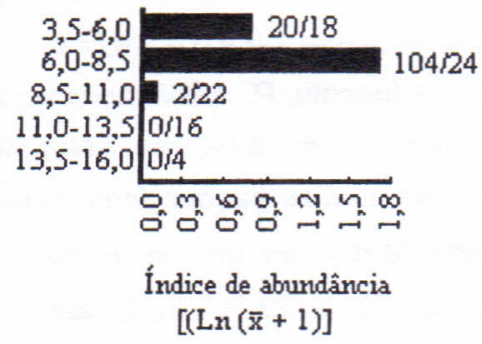

Índice de abundância $[(\operatorname{Ln}(\bar{x}+1)]$

Figura 2: Abundância de Portunus spinimanus Latreille, 1819 em função dos fatores: temperatura, salinidade, oxigênio dissolvido, matéria orgânica do sedimento e profundidade. 


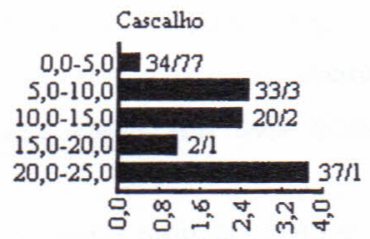

Areia Média

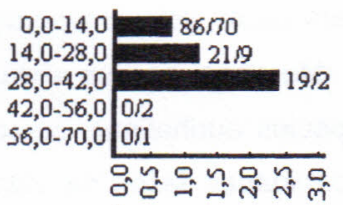

Índice de abundância $[(\operatorname{Ln}(\bar{x}+1)]$
Areia Muito Grossa

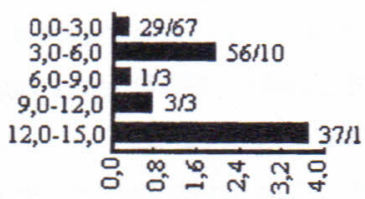

Areia Fina

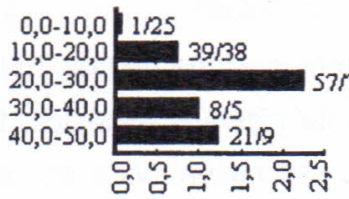

Silte-Argila

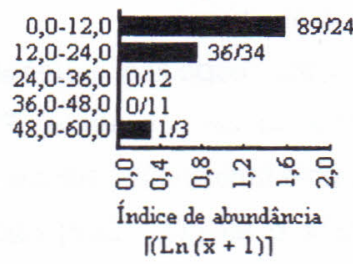

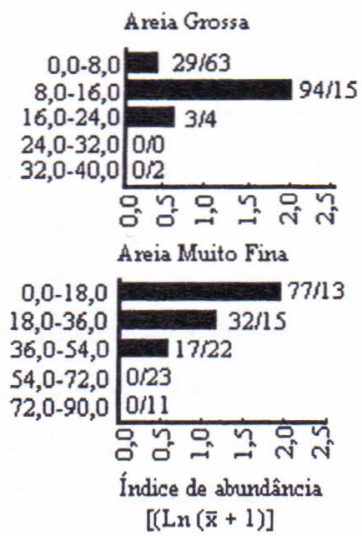

$[(\operatorname{Ln}(\bar{x}+1)]$

Figura 3: Abundância de Portunus spinimanus Latreille, 1819 em função das frações granulométricas do sedimento: cascalho, areia muito grossa, areia grossa, areia média, areia fina, areia muito fina e silte-argila. 


\section{DISCUSSÃO}

A temperatura, provavelmente, não constitui fator determinante exclusivo na abundância de $P$. spinimanus na Enseada da Fortaleza, pois as variações registradas foram mínimas de subárea para subárea. O maior índice de abundância deveu-se principalmente à presença de jovens no final do verão. Segundo WILLIAMS (1984) esta espécie vive em locais com temperatura variando entre 19,2 a $32^{\circ} \mathrm{C}$.

Mesmo em áreas limitadas, como é o caso de uma enseada, observa-se variações de salinidade de um local para outro, principalmente próximo a regiões de desembocadura de rios. Estas diferenças podem afetar o modelo de distribuição de algumas espécies. Espécies eurihalinas, como Callinectes danae Smith, são encontradas em determinadas fases da vida em ambientes de baixa salinidade e em outras, em ambientes com salinidade superior (PITA et al., 1985).

Por outro lado, espécies estenohalinas distribuem-se em ambientes com baixa variação de salinidade. $P$. spinimanus enquadra-se nesta segunda categoria, pois tanto neste estudo como em outros (CAMP et al., 1977); WILLIAMS, 1984 e MELO, 1996) não se registrou a presença desta espécie em estuários. No entanto, isto não é regra para o gênero porque $P$. pelagicus, estudado por POTTER et al. (1983) ocorre em águas de baixa salinidade.

O teor de oxigênio dissolvido na água apresentou-se sempre próximo do ponto de saturação. $O$ fato de um maior número de exemplares ser capturado na faixa de 4,60 a 5,70 mg/L, sugere uma baixa demanda de oxigênio de $P$. spinimanus. GRAY (1957) afirma que a área branquial de $P$. spinimanus é intermediária entre as espécies Arenaeus cribrarius (Lamarck), C. sapidus, Ovalipes ocellatus ocellatus (Herbst) e Portunus gibesii (Stimpson), o que sugere exigências moderadas quanto à disponibilidade de oxigênio. 
Estes resultados aliado a outros obtidos por DHAWAN et al. (1976) e BUCHANAN \& STONER (1988) para outros portunídeos confirmam que alguns siris, capturados em ambientes com moderados teores de oxigênio, provavelmente adaptam-se bem à estas condições.

A profundidade, a composição granulométrica do sedimento e seu teor de matéria orgânica são outros fatores que variam significativamente nesta área (NEGREIROS-FRANSOZO et al., 1991). A profundidade, via topografia, exerce grande influência no padrão granulométrico de ambientes específicos (MAGLIOCCA \& KUTNER, 1965). A literatura menciona que $P$. spinimanus distribui-se até $90 \mathrm{~m}$ de profundidade (WILLIAMS, 1984; MELO, 1996), porém os resultados evidenciam maior abundância desta espécie, na enseada, nas profundidades de 6,0 a 8,5 m. Com a análise da distribuição das categorias etárias, verificou-se que tal abundância deveu-se ao elevado número de jovens próximo a Praia do Lázaro onde a profundidade varia de 04 a 08 metros (NEGREIROSFRANSOZO et al., 1991). A presença de indivíduos jovens em ambientes mais rasos está em concordância com os registros de outros autores (DUDLEY \& JUDY, 1971; NORSE \& ESTEVEZ, 1977 e PAUL, 1982).

Locais com alto teor de matéria orgânica e silte-argila podem refletir maior quantidade de alimentos disponíveis para espécies bentônicas (MAGLIOCCA \& KUTNER, 1965). Este pode ser um fator importante relacionado com a presença de $P$. spinimanus, principalmente para os jovens. Mesmo que estes animais não se alimentem diretamente da matéria orgânica, esta pode servir como alimento para suas presas, uma vez que os portunídeos são omnívoros, com tendência à carnívoros, de acordo com WILLIAMS (1982) e NONATO et al. (1990).

Animais detritivoros normalmente vivem em locais onde a quantidade de matéria orgânica é mais elevada. Os resultados obtidos evidenciam que $P$. spinimanus foi mais abundante na faixa entre 5,0 e 7,5\%, teores encontrados próximo à praia do Lázaro (Subárea 2). No entanto, $P$. 
spinimanus não ocorreu na boca e no meio da enseada, onde foram registrados teores que não diferiram significativamente da'subárea 2. Desta forma é bastante provável que o conteúdo de matéria orgânica no sedimento não esteja diretamente ligado à presença de $P$. spinimanus na enseada. Além disso, NONATO et al. (1990) relatam que os anelídeos demonstraram ser um item muito importante na dieta dos decápodos braquiúros e $P$. spinimanus alimenta-se de animais tipicamente sésseis como os depositivoros sésseis de superfície (Familia Terebellidae) e os suspensívoros (Família Serpulidae), não apresentando o hábito de cavar à procura de alimento.

A abundância de $P$. spinimanus não pode ser atribuída a um único fator ambiental, porém, pode-se inferir, baseando-se nos resultados aqui apresentados, que esta espécie está associada a um conjunto de fatores ambientais, entre os quais destacam-se a textura do sedimento (cascalho e areia muito grossa) e teor moderado de oxigênio.

\section{AGRADECIMENTOS}

À Fundação de Amparo à Pesquisa do Estado de São Paulo (FAPESP), pelo auxílio financeiro (Proc. $n^{\circ}$ 92/3623-0). Aos colegas do NEBECC (Núcleo de Estudos em Biologia, Ecologia e Cultivo de Crustáceos), pela colaboração nas coletas de campo e nas análises de laboratório. 


\section{REFERÊNCIAS BIBLIOGRÁFICAS}

BUCHANAN, B. A.; STONER, A. W. Distribution patterns of blue crabs (Callinectes $s p$.) in a tropical estuarine lagoon. Estuaries, v. 11, n.4, p. 231-239, 1988.

CAMP, D. K.; WHAITING, N. H.; MARTIN, R. E. Nearshore marine ecology at Hutchinson Island Florida 1971-1974. V. Arthropods. Fla. Mar. Res. Publ., v. 25, p.1-63, 1977.

DHAWAN, R. J.; DWIVED, S. N.; RAJAMANICKAM, G. V. Ecology of the blue crab Portunus pelagicus (L.) and its potential fishery in Zuari Estuary. Indian J. Fish., v. 23, n. 1/2, p.57-64, 1976.

DUDLEY, D. L.; JUDY, M. H. Occurrence of larval, juvenile, and mature crabs in the vicinity of Beaufort Inlet, North Carolina. NOAA Tech. Rep. NMFS/SSRF, v. 637, p.1-10, 1971.

GOLTERMAN, H. L.; CLYMO, R. S. Methods for chemical analysis of freshwaters. Oxford: Blackwell, Scientific Publications, 1969. 116p.

GRAY, I. E. A. comparative study of the gill area of crabs. Biol. Bull., v. 112, n.1, p.34-42, 1957.

HAEFNER JR., P.A. Distribution, reproductive and molting of the rock crab, Cancer irroratus Say, 1917, in the mid-Atlantic bigh. J. Nat. Hist., v. 10, p.377-397, 1976.

MAGLIOCCA, A. ; A S. KUTNER. Sedimentos de fundo da Enseada do Flamengo - Ubatuba. Publ. n. 198 do Inst. Ocean. da USP. P. 1-14, 1965.

MELO, G. A. S. Manual de identificação dos Brachyura (caranguejos e siris) do litoral brasileiro. São Paulo: Editora Plêiade, 1996. 603p.

NEGREIROS-FRANSOZO, M. L.; FRANSOZO, A.; PINHEIRO, M. A. A.; MANTELATTO, F. L. M.; SANTOS, S. Caracterização física e química da Enseada da Fortaleza, Ubatuba, SP. Rev. Bras. Geoc., v. 21, n. 2, p.114120, 1991.

NONATO, E. F.; PETTI, M. A. V.; PAIVA, P. C. Contribuição dos anelídeos poliquetas na dieta de crustáceos decápodos braquiúros da região de Ubatuba. In: SIMPÓSIO DE ECOSSISTEMAS DA COSTA SUL E SUDESTE BRASILEIRA, Águas de Lindóia, 1990. Anais... São Paulo: Academia Paulista de Ciências, v. 1, p.224-234. 
NORSE, E. A.; ESTEVEZ, M. Studies on portunid crabs from the eastern Pacific. I. Zonation along environment stress gradients from the coast of Colombia. Mar. Biol., v. 40, p. 365-373, 1977.

PAUL, R. K. G. Observations on the ecology and distribution of swimming crabs of the genus Callinectes (Decapoda, Brachyura, Portunidae) in the gulf of California, Mexico. Crustaceana, v. 42, n. 2, p.96-100, 1982.

PITA, J. B.; RODRIGUES, E. S.; GRAÇA-LOPES, R.; COELHO, J. A. P. Observações bioecológicas sobre o siri Callinectes danae Smith, 1869 (Crustacea, Portunidae) no complexo baía-estuário de Santos, Estado de São Paulo, Brasil. Bol. Inst. Pesca, v. 12, n. 4, p.35-43, 1985.

POTTER, I. C.; CHRYSTAL, P. J.; HONERAGAN, N. The biology of the blue manna crab Portunus pelagicus in a Australian estuary. Mar. Biol., v. 78, p.75-85, 1983.

POWERS, L. W. Crabs (Brachyura) of the gulf of the Mexico. Contr. Mar. Sci. (Supl.), v. 20, p.1-190, 1977.

TAISSOUN, E. N. Biogeografía y ecologia de los cangrejos de la familia Portunidae (Crustáceos, Decápodos, Brachyura) en la costa Atlântica de América. Boln. Centr. Invest. Biol. Univ. Zulia, Maracaibo, v. 7, p.7-23, 1973a.

TAISSOUN, E. N. Las especies de cangrejos del genero Callinectes (Brachyura) en el golfo de Venezuela y Lago de Maracaibo. Boln. Cent. Invest. Biol. Univ. Zulia Maracaibo, v. 2, p.1-102, 1973b.

WENTHWORTH, C. K. A scale of grade and class terms for clastic sediments. J. Geol., v. 30, p.377-392, 1922.

WILLIAMS, A. B. Shrimps, lobsters and crabs of the Atlantic Coast of the eastern United States, Maine to Florida. Washington: Smithsonian Institution Press, 1984. 550p.

WILLIAMS, M. J. Natural food and feeding in the commercial sand crab Portunus pelagicus (L. 1766) (Crustacea, Decapoda, Portunidae) in Moreton Bay, Queensland. J. Exp. Mar. Biol. Ecol., v. 59, p.165-179, 1982. 
Tabela I: Valores médios e desvio padrão dos dados dos fatores ambientais registrados durante as coletas (nov/88 a out/89).

\begin{tabular}{|c|c|c|c|c|c|}
\hline Fatores & Temperatura $\left({ }^{\circ} \mathrm{C}\right)$ & Salinidade (\%o) & Oxigênio (mg/L) & Matéria Orgânica (\%) & Profundidade $(\mathrm{m})$ \\
\hline \multicolumn{6}{|l|}{ Subáreas } \\
\hline 1 & $22,6 \pm 2,3$ & $34,8 \pm 0,8$ & $5,61 \pm 0,9$ & $4,42 \pm 2,5$ & $11,2 \pm 0,9$ \\
\hline 2 & $23,7 \pm 2,6$ & $34,3 \pm 1,3$ & $5,41 \pm 0,9$ & $6,65 \pm 2,4$ & $2,96 \pm 0,9$ \\
\hline 3 & $23,8 \pm 2,5$ & $34,4 \pm 1,1$ & $5,28 \pm 1,1$ & $2,32 \pm 1,3$ & $8,46 \pm 0,9$ \\
\hline 4 & $24,4 \pm 2,6$ & $33,3 \pm 1,5$ & $5,86 \pm 1,4$ & $1,84 \pm 1,3$ & $4,42 \pm 0,6$ \\
\hline 5 & $23,4 \pm 2,1$ & $34,4 \pm 1,1$ & $6,10 \pm 0,8$ & $3,54 \pm 1,4$ & $7,13 \pm 0,8$ \\
\hline 6 & $23,8 \pm 2,6$ & $34,4 \pm 1,1$ & $4,95 \pm 1,3$ & $5,16 \pm 1,8$ & $11,1 \pm 1,2$ \\
\hline 7 & $22,7 \pm 2,4$ & $34,9 \pm 1,7$ & $4,99 \pm 1,3$ & $4,56 \pm 3,6$ & $13,3 \pm 1,6$ \\
\hline
\end{tabular}


Tabela II: Enseada da Fortaleza, Ubatuba, SP. Composição granulométrica: valores médios (em gramas) obtidos em cada subárea, analisados pelo método de Tukey $(P<0,01)$. C (Cascalho), AMG (Areia Muito Grossa), AG (Areia Grossa), AM (Areia Média), AF (Areia Fina), AMF (Areia Muito Fina), SA (Silte Argila).

\begin{tabular}{cccccccc}
\hline Frações & $\mathrm{C}$ & $\mathrm{AM} \mathrm{G}$ & $\mathrm{A} \mathrm{G}$ & $\mathrm{AM}$ & $\mathrm{AF}$ & $\mathrm{AMF}$ & $\mathrm{S} \mathrm{A}$ \\
\hline Subáreas & & & & & & & \\
1 & $0,18 \mathrm{bc}$ & $0,93 \mathrm{~b}$ & $3,81 \mathrm{a}$ & $5,55 \mathrm{a}$ & $2,58 \mathrm{~d}$ & $10,01 \mathrm{e}$ & $6,94 \mathrm{c}$ \\
2 & $1,73 \mathrm{a}$ & $1,37 \mathrm{a}$ & $2,45 \mathrm{~b}$ & $4,72 \mathrm{~b}$ & $5,62 \mathrm{~b}$ & $6,73 \mathrm{f}$ & $7,38 \mathrm{c}$ \\
3 & $0,02 \mathrm{c}$ & $0,27 \mathrm{ce}$ & $0,59 \mathrm{~d}$ & $0,72 \mathrm{f}$ & $3,59 \mathrm{c}$ & $16,60 \mathrm{~b}$ & $8,21 \mathrm{~b}$ \\
4 & $0,01 \mathrm{c}$ & $0,01 \mathrm{~d}$ & $0,05 \mathrm{e}$ & $0,30 \mathrm{~g}$ & $2,90 \mathrm{~d}$ & $22,27 \mathrm{a}$ & $4,46 \mathrm{~d}$ \\
5 & $0,22 \mathrm{~b}$ & $0,20 \mathrm{de}$ & $0,66 \mathrm{~d}$ & $2,01 \mathrm{~d}$ & $11,14 \mathrm{a}$ & $11,67 \mathrm{~d}$ & $4,10 \mathrm{~d}$ \\
6 & $0,08 \mathrm{bc}$ & $0,45 \mathrm{c}$ & $2,27 \mathrm{~b}$ & $3,09 \mathrm{c}$ & $5,33 \mathrm{~b}$ & $9,55 \mathrm{e}$ & $9,23 \mathrm{a}$ \\
7 & $0,04 \mathrm{bc}$ & $0,39 \mathrm{ce}$ & $1,39 \mathrm{c}$ & $1,69 \mathrm{e}$ & $3,62 \mathrm{c}$ & $14,58 \mathrm{c}$ & $8,29 \mathrm{~b}$ \\
\hline
\end{tabular}

* As médias seguidas de pelo menos uma letra em comum, na coluna, não diferem entre si. 